\title{
Lactate as a Predictor in Severe Pneumonia
}

\author{
Darta Ose*, Arvids Berzins**, Krista Grigorovica***, Andris Klucniks****, Olegs Sabelnikovs***** \\ *Riga Stradins university, medical faculty \\ ** Mayo General Hospital, Ireland \\ *** Our Laidy's Children Hospital, Crumlin, Ireland \\ **** Oxford University Hospitals NHS Trust, United Kingdom \\ ***** Riga Stradins University, Department of Anesthesiology and Resuscitation; Pauls \\ Stradins Clinical University Hospital; Latvia
}

\section{SUMMARY}

Introduction. There are many discussions that one of the mortality risk markers for patients admitted to intensive care unit (ICU) with different etiology shock is admission blood lactate level. It is believed that serum lactate could be used as an early marker of mortality risk determination. We are not aware about research studies regarding admission serum lactate prognostic significance in patients with severe community acquired pneumonia (CAP) in first 24 hours after admission to ICU in Latvia.

\section{Aim of the study.}

1. To evaluate the prognostic significance of the first lactate level in patients with severe pneumonia. To compare the statistical data of different blood lactate levels $\left(\mathrm{LAC}_{1}, \mathrm{LAC}_{2}, \mathrm{LAC}_{3}, \mathrm{LAC}_{4}, \mathrm{LAC}_{\mathrm{MAX}}, \mathrm{LAC}_{\mathrm{MIN}}\right)$.

2. To find admission lactate reference level which is attributed to significant increase of mortality.

3. To compare with other markers and scoring systems like: PCT (procalcitonin), CRP (C-reactive protein), CURB-65 (pneumonia severity score), APACHEII (Acute Physiology and Chronic Health Evaluation II).

Material and methods. This is a retrospective observational study in which data were collected on all patients admitted to ICUwith pneumonia and sepsis and/or septic shock in two major Hospitals of Republic of Latvia (Eastern Clinical university hospital and Pauls Stradins Clinical university hospital) with primary diagnosis of severe community acquired pneumonia (CAP). We compared the relationship between lactate values that were collected in 24 hour period after admission in ICU and ICU mortality.

Results. In this study we analyzed data from consecutive 73 patients with severe CAP and sepsis and/or septic shock and we observed statistically significant difference between the first lactate level (LAC $)$ in survivors (2.7 [1.9-3.2] (mmol/l) and nonsurvivors 4.9 [4.3-7.5] (mmol/l); $p<0.001)$. According to data patients withLAC $<3.0(\mathrm{mmol} / \mathrm{l})$ mortality risk was $0 \%$, patients with $\mathrm{LAC}_{1} 3.0-4.0$ (mmol/l) risk was $42.1 \%$, while patients with $\mathrm{LAC}_{1}>4.0$ (mmol/l) mortality risk reached $89.7 \%$. Lactate level measurements in first 24 hours after arrival into the ICU have had high ability to stratify non-survivor patients: $L A C_{1}(0.96)$, LAC (0.98), $\mathrm{LAC}_{3}(0.97), \mathrm{LAC}_{4}(0.92)$, (AUC). Incomparison with other prognostic markers sensitivity and specificity following results were obtained: CRP (0.59), PCT (0.98), APACHE II (0.98), CURB-65 (0.63).

Conclusions. Summarizing data on patients with severe pneumonia and sepsis and/or septic shock admission lactate in first 24 hours have significant independent predictive value. In first 24 hours after admission in ICU higher mortality were observed if LAC was $>3(\mathrm{mmol} / \mathrm{l})$. Data proves for thepatients with severe pneumonia $\mathrm{LAC}_{1}$ is having similar prognostic ability like APACHEII and PCT, and significantly better prognostic ability than CRP and CURB-65.

Key words: admission lactate; intensive care unit; severe pneumonia; mortality

\section{INTRODUCTION}

According to data collected at 2010 for total mortality and causes of death in the world, lower respiratory tract infections are the cause of death in 2.8 million cases from 52.8 million. (Lozano, 2012). Several sources show that $20-36 \%$ of patients with pneumonia requires hospitalization in ICU andfor them the risk of the mortality is above $70 \%$ (Wiemken, 2012; Gutierrez, 2005; Peake, 2014; Moine, 1994).

For critically ill patients with a different reasons for shock high lactate levels at the beginning are associated with higher mortality risk (Gunnerson, 2006; Mikkelsen, 2009; Suistomaa, 2000).Lactateis the end product of anaerobic glycolysis and for most patients with septic shock it rises due to tissue ischemia and insufficient oxygen supply. One of the most recent studies indicates that the starting lactate levels $\geq 4(\mathrm{mmol} / \mathrm{l})$ is associated with a high risk of mortality for trauma patients (Odom, 2014).
The prognostic importance of the initial lactate levels in critically ill patients with a different shock etiologies were showed in one of the first studies about lactate levels. This study indicated that for those patients who had their lactate levels $>3.83 \mathrm{mmol} / \mathrm{l}$ they were associated with $67 \%$ mortality, while those who had $<3.83$ (mmol/l), with only $25 \%$ mortality rate.(Cady, 1973). Increase in lactate level can be observed even before any significant changes in the vital indicators. (Bernardin, 1996; Shapiro, 2005). Moreover, early detection gives a broader assessment of the patientcondition and the opportunity of an early initiation of adequate treatment, which reduces in-hospital mortality rate (Jansen, 2010). Surviving Sepsis guidelines, updated in 2012, points out the fact that serum lactate level is a very important indicator for the severity of sepsis. It recommends to measure lactate serum levels in the first three hours after admission and to achieve its normalization as quickly as possible (Dellinger, 2013). 
Currently it is not clear what is the initial lactate level that would show a particularly high risk of mortality in patients with severe pneumonia. And how it is related to prognosis in ICU. For better prognostic significance in lactate level analysis, it was compared with such markers as PCT, CRP, CURB-65, APACHE II.

\section{AIM OF THE STUDY}

1. To evaluate the prognostic significance of the first lactate level in patients with severe pneumonia. To compare the statistical data of $\left(\mathrm{LAC}_{1}, \mathrm{LAC}_{2^{\prime}} \mathrm{LAC}_{3^{\prime}}\right.$ $\mathrm{LAC}_{4^{\prime}} \mathrm{LAC}_{\mathrm{MAX}^{\prime}}, \mathrm{LAC}_{\mathrm{MIN}}$ ).

2. To find $L A C$ reference level which is attributed to significant increase of mortality.

3. To compare with other markers and scoring systems like: PCT (procalcitonin), CRP (C-reactive protein), CURB-65 (pneumonia severity score), APACHEII (Acute Physiology and Chronic Health Evaluation II).

\section{MATERIAL AND METHODS}

This is a retrospective study. In this study patient medical charts were collected form records found in hospital archives.Included data were collected from the ICU of the two major hospitals in Republic of Latvia - Eastern Clinical University Hospital(ECUH) and Pauls Stradins Clinical University Hospital(PSCUH). Conduction of the study were aligned with the Riga Stradins university (RSU) Ethics Committees Data, which meet the criteria set out in the study, were collected from January 2012 to May 2014.

In the study were included patients who were admitted to ICU with severe pneumonia with sepsis and/or septic shock and arterial blood gases were taken in ICU at least twice in the first 24 hours. Patients with fixed other serum level measurements: CRP, procalcitonin, leucocytes, hematocrit and creatinine in first day. And with documented level of consciousness after the Glasgow coma scale, respiratory rate, heart rate and blood pressure. Patients with lung cancer, active lung tuberculosis, HIV and those who were taken medications of the biguanide group were excluded from this study. In total 543 medical charts were reviewed. 470 of those were excluded from the study because on the first admission day they spent in other ward or there were lack of data and misleading diagnosis. Rest of the charts - 73 of them where included in the study. For the collection of the data were used study protocol (appendix 1). Data were analyzed with MS Excel and SPSS programmes. Statistical changes between groups of subjects and variables were analyzed by KolmogorovSmirnov Test, Mann-Whitney $U$ test, t-test. Where Statistically significant results are with $p<0,05$. Whereas with ROC (Reciever-Operating Characteristic) curves results were measured for various prognostic markers of accuracy regardless of the defined threshold, taking into account the sensitivity and specificity of the markers. Data for normal distribution groups were processed by parametric statistical analysis techniques, but for data that doesn't meet the normal distribution criteria were processed by non-parametric statistical methods.

After discharge from the ICU selected patients were divided into two groups: survivors and non-survivors. Among these groups lactate levels were in the first 24 hours. Also prognostic significance were compared with other severe pneumonia and shock prognostic markers (PCT, CRP) and scales (CURB-65, APACHE II).

Included patients with diagnosis of severe pneumonia were determined by SIRS criteria (Members of the American College of Chest Physicians/Society of Critical Care Medicine Consensus Conference Committee, 1992), where patients who has been diagnosed with pneumonia and have $\geq 2$ of the following clinical findings: fever $>38,3^{\circ} \mathrm{C}$ or hypothermia $<36^{\circ} \mathrm{C}$, leukocytosis $>12$ $(10 \wedge 9 / \mathrm{L})$ or leukopenia $<4(10 \wedge 9 / \mathrm{L})$ or normal white blood cell count with more than $10 \%$ of the new form, tachycardia $>90$ ( $\mathrm{x} / \mathrm{min}$ ), tachypnea $>20$ (x / min) or PaCO2 <32 (mmHg). CURB-65 and APACHE II scores were applied for more accurate evaluation of the patient's condition. Co-morbidities, as a risk factor influencing mortality rate were evaluated with APACHE II scale, that includes 12 routinely used markers and it is recognized as the most objective scale for patients with acquired pneumonia (Loke, 2010; Neil, 1996; Knaus, 1985; Richards, 2011).

\section{RESULTS}

\section{Characteristics of the study population}

Medical records were analyzed for 73 ICU patients who were hospitalized in the intensive care unit due to relative hypovolemia and respiratory failure.

The mean age of patients was $63.2( \pm 16.6)$ years. The study included 41 male and 32 female.34 (48.6\%) patients died in the ICU with a mean age of $69.7( \pm 16.6)$, including $14(41.2 \%)$ women and $20(58.8 \%)$ men. Of these 34 deaths, the youngest patient was 28 years old whereas the oldest had 93 years.Characteristicsof the two groups are seen in Table 1. Lactate level analysis was done with Mann-Whitney U test. The results are seen in Table 2. 
Table 1. Characterization of Non-survivor and survivor groups

\begin{tabular}{|l|c|c|c|l|}
\hline & Survivors (39) & Non-survivors (34) & P value & Test \\
\hline Demographic data & & & & \\
\hline Age & $57,5( \pm 14,4)$ & $69,7( \pm 16,6)$ & 0,001 & t-test \\
\hline Sex, female $(\%)$ & $18(46,2 \%)$ & $4(41,2 \%)$ & 0,69 & Chi square \\
\hline Characteristics of groups & & & & \\
\hline Septic shock & $14(35,9 \%)$ & $22(64,7 \%)$ & 0,001 & Chi square \\
\hline APACHE II & $14(12-16)$ & $25(21-30)$ & 0,001 & Mann-Whitney U test \\
\hline CURB-65 & $3(2-4)$ & $3(3-4)$ & 0,34 & Mann-Whitney U test \\
\hline Leu (10^9/L) & $13(10-15)$ & $3,5(2-4)$ & 0,001 & Mann-Whitney U test \\
\hline CRP (mg/l) & $230(104-347)$ & $268(170-450)$ & 0,14 & Mann-Whitney U test \\
\hline PCT (ng/ml) & $9,0(5,7-14,0)$ & $91,0(62-159)$ & 0,001 & Mann-Whitney U test \\
\hline MAP (mmHg) & $71,7(69,7-82)$ & $65(54,7-53)$ & 0,001 & Mann-Whitney U test \\
\hline PaO2/FiO2 & $151(151-114)$ & $103(76-127)$ & 0,001 & Mann-Whitney U test \\
\hline Lenght of stay in ICU & & & & \\
\hline days & $8(5-14)$ & $5(2-10)$ & 0,006 & Mann-Whitney U test \\
\hline
\end{tabular}

A chronic co-morbid conditions were recorded among 7 dead patients: 2 with chronic obstructive pulmonary disease (COPD), 2 with immunosuppression, 1 with chronic heart failure (CHF), 1 with CHFand immunosuppression and 1 with chronic renal failure. Among survivor group 2 where with COPD, 1 with CHF and 1 with immunosuppression.

Table 2. Serum lactate concentration levels in survivor and non-survivor groups

\begin{tabular}{|l|c|c|l|}
\hline & Survivors & Non-survivors & P value \\
\hline Static lactate level indices & & & \\
\hline $\mathrm{LAC}_{\text {max }}$ & $3(2-3)$ & $6(5-8)$ & 0,001 \\
\hline $\mathrm{LAC}_{\min }$ & $2(2-2)$ & $4(3-5)$ & 0,001 \\
\hline $\mathrm{LAC}_{1}$ & $2,7(1,9-3,2)$ & $4,9(4,3-7.5)$ & 0,001 \\
\hline $\mathrm{LAC}_{2}$ & $2,0(1,7-2,4)$ & $4,0(4,0-6,0)$ & 0,001 \\
\hline $\mathrm{LAC}_{3}$ & $2,0(1,9-2,0)$ & $4,0(2,9-5,6)$ & 0,001 \\
\hline $\mathrm{LAC}_{4}$ & $2,0(2,0-2,0)$ & $3,3(2,3-4,8)$ & 0,048 \\
\hline
\end{tabular}

\section{The obtained serum lactate values}

The first measurement of serum lactate $\left(\mathrm{LAC}_{1}\right)$ was carried out in the first hour of

hospitalization in the ICU. The study showed statistically significant differences between survivors and deceased patients in first and all subsequent measurements (second, third and fourth). The biggest difference in lactate median values between survivors and deceased patients were observed in the first measurement, and over time the difference tended to decrease. The maximum and minimum median of lactate levels for surviving patients was lower than that of the not survivors. Patients who LACl level were 3.0 to 4.0 (mmol/ l) the mortality risk was $42.1 \%$, while if LACl level was $>4.0$ (mmol / l) mortality risk was $89.7 \%$ (see. Table 3 ).

Table 3. Mortality risk according to first serum lactate measurement

\begin{tabular}{|l|l|}
\hline LAC $_{1}$ level $(\mathrm{mmol} / \mathrm{l})$ & Risk of mortality $(\%)$ \\
\hline $1-2$ & 0 \\
\hline $2-3$ & 0 \\
\hline $3-4$ & 42,1 \\
\hline$>4$ & 89,7 \\
\hline
\end{tabular}

In the group of not survivors, $26(76 \%)$ of patients had LAC $1 \geq 4,5$ (mmol/l), and these patients average length of stay in the ICU was 3.8 days. In addition, seven of them died in the first day, 13 patients during the first three days and 22 in during the first week. While if LACl was $>8$ (mmol / l), which was registered in 6 dead patients, the average number of days spent in the ICU had dropped to 2.16 days.

\section{Dynamics of lactate level measurements}

Second lactate level measurements for non-survivors are performed earlier than in survivors, respectively after four hours (3-7) in non-survivor group and after six hours(5-9) in survivors group $(p=0.007)$.Interval of time in hours (h) between the second and third lactate level $\left(\mathrm{LAC}_{2-3}\right)$ as well as third and fourth measurement $\left(\mathrm{LAC}_{3-4}\right)$ is similar to the non-survivor and to survivor patient groups.

Table 4. Dynamics of lactate level measurements in the first 24 hours

\begin{tabular}{|l|c|c|c|c|c|c|c|c|c|c|c|c|c|c|c|c|c|c|c|c|c|c|c|c|}
\hline & 1 & 2 & 3 & 4 & 5 & 6 & 7 & 8 & 9 & 10 & 11 & 12 & 13 & 14 & 15 & 16 & 17 & 18 & 19 & 20 & 21 & 22 & 23 & 24 \\
\hline Survivors & $\mathbf{x}$ & - & - & - & - & $\mathbf{x}$ & - & - & - & - & - & - & $\mathrm{x}$ & - & - & - & - & - & - & - & - & - & $\mathrm{x}$ & - \\
\hline Non-surviv. & $\mathrm{x}$ & - & - & $\mathrm{x}$ & - & - & - & - & - & - & $\mathrm{x}$ & - & - & - & - & - & - & $\mathrm{x}$ & - & - & - & - & - & - \\
\hline
\end{tabular}


Characteristics of lactate level discriminatory capacityof the survival prognosis in ICU

ROC curves were made and area under the curve (AUC) were compared with lactate concentration characteristics (Tab 5) in order to assess the predictive ability of lactate level to separate survivor group from not survivors for ICU patients with severe pneumonia during the first 24 hours.

Table 5.Serum lactate level discriminatory ability

\begin{tabular}{|l|l|}
\hline & AUC \\
\hline$L A C_{1}$ & 0,96 \\
\hline$L A C_{2}$ & 0,98 \\
\hline $\mathrm{LAC}_{3}$ & 0,97 \\
\hline $\mathrm{LAC}_{4}$ & 0,92 \\
\hline $\mathrm{LAC}_{\max }$ & 0,98 \\
\hline $\mathrm{LAC}_{\min }$ & 0,91 \\
\hline
\end{tabular}

\section{Other prognostic markers}

The results show that all the lactate measured during the first 24 hours is a good prognostic marker (Fig. 2). Inaccurate markers are CRP (0.60), CURB-65 (0.63), but significantly more accurate PCTs (0.98) APACHEII (0.98) LACl (0.96) (Fig 1). White blood cell count $<3$ $(10 \wedge 9 / 1)$ are noted for 16 patients, but $>15(10 \wedge 9 / 1)$ in one patient in not survived patient group. Surviving patients, respectively, leukocytosis had five patients, but leukopenia nil.

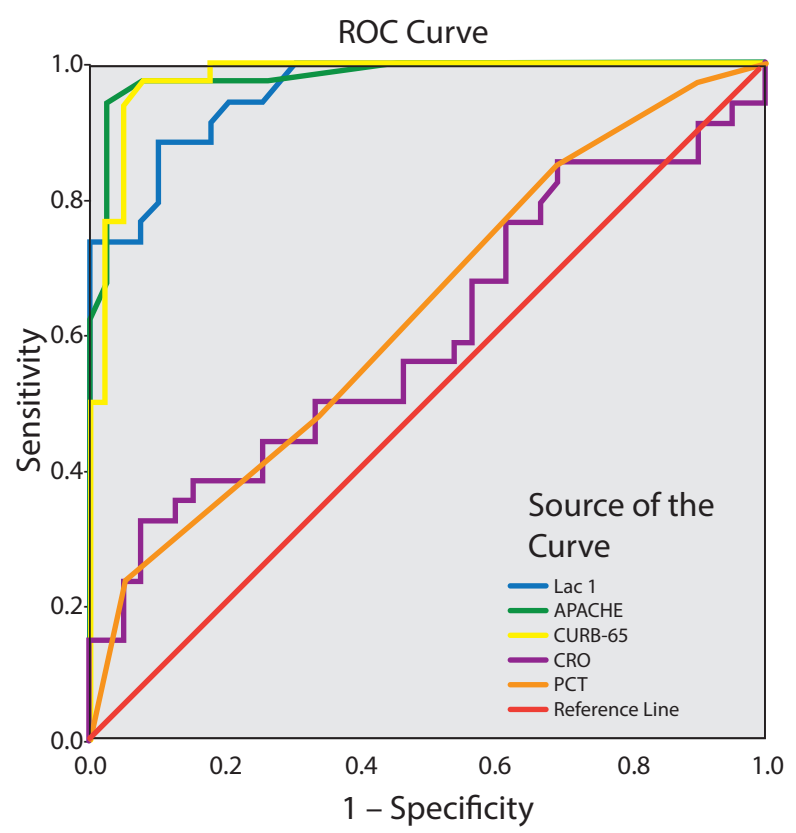

Diagonal segments are produced by ties.

Fig. 1. Initial serum lactate level comparison with other prognostic factors

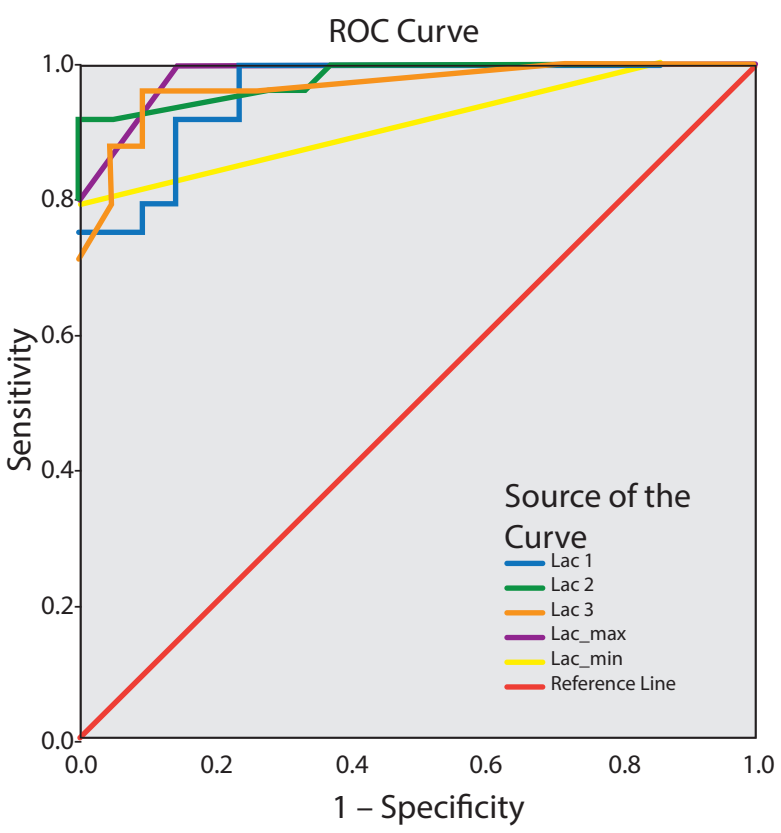

Diagonal segments are produced by ties.

Fig. 2 Static serum lactate comparison of sensitivity and specificity

\section{DISCUSSION}

The study retrospectively analyzed 73 patients with severe pneumonia. After they were admitted to ICU lactate level was measured 2-4 times to determine lactate levels in the blood. The aim was to investigate whether initial lactate levels are an important prognostic indicator of mortality in patients with severe pneumonia during the first 24 hours. The study defined sepsis as a verified or expected infection with $\geq 2$ SIRS criteria, that is accepted to use in the diagnosis of sepsis (Dellinger, 2013; Members of the American College of Chest Physicians/Society of Critical Care Medicine Consensus Conference Committee, 1992). There is a presumption that in the case of septic shock lactate level elevations are associated with multifactorial etiology, however, it is demonstrated that lactate levels for critically ill patients with septic shock is a good warning signal for the early and effective start of treatment (Rivers, 2001). Chertoff et al study, collected data show that lactate kinetics is particularly prognostic significance directly early stages of sepsis (Chertoff, 2015). It is therefore important to determine lactate levels as quickly as possible. It could be considered as a routine practice to check for lactate level in venous blood before hospitalization to ICU. This will accelerate early and effective treatment initiation in patients with severe pneumonia. In this study for patients with severe pneumonia and $\mathrm{LAC}_{1}>4(\mathrm{mmol} / \mathrm{l})$, there is a significant increase in mortality risk. Lactate level could be used as atool for initiation of effective therapy in patients with severe pneumonia and closer 
(most likely invasive) monitoring and higher levels of care. In addition, surviving patients had no one who LAC $_{1}>4.5(\mathrm{mmol} / \mathrm{l})$. The best ability to distinguish potentially non-survivors LAC $_{\text {MAX }}$ patients was observed, however, the maximum lactate in the first 24 hours is not necessarily the first measurement of lactate, which limits the role of the forecast already at the time of hospitalization. The best prognosis was observed if $\mathrm{LAC}_{1}$ was 2-4 (mmol/l). Out of all these patients a more favorable prognosis were associated with lower initial lactate level. Some patients may not always require admission to the ICU, if their lactate levels were $>4$ $(\mathrm{mmol} / \mathrm{l})$, and not requiring respiratory, cardiovascular or any other organ support. Regardless of the time lactate measurement were taken in the first 24 hours, it is a good prognostic marker. In comparison with the other characteristics LAC is similar to PCT and APACHE II. For APACHE II scale need quite a lot of characteristics and PCT quantification is longer and more expensive, so practically lactate is easier to use. It is possible that patients in non-survivor group from the very beginning were presenting with more severe clinical condition and that is why these patients had higher lactate levels in the first place and good results with "correct" treatment was harder to reach. Due to the fact that patients therapy was not analyzedit is impossible to evaluate effectiveness of therapy used in each case. Etiological agent was not analyzed because approximately in half of the cases it was not found which is similar to data from other scientific papers (Peake, 2014). In this research authors wanted to emphasize the significance of metabolic factors independently from etiological agent, which in most cases is not identified in the first 24 hours. We analyzed patients with Community Acquired Pneumonia, which is why the results of the study cannot be associated with other etiology pneumonias like Hospital-acquired Pneumonia, Health care-acquired pneumonia, Aspiration pneumonia. In this study medications that patients might had received at home before hospitalization were not analyzed. This can influence a serum lactate levels, like metformin, epinephrine or any other sypathomimetics and other hyperlactataemia inducing agents. Although chronic heart failure can chronically increase lactate levels, two patients were included in the research that does not change the general results and conclusions. Also in this study we did not find a statistically reliable correlation between CURB-65 and the outcome. That can be explained with the fact that average age of patients in this study was under 65 , respiratory rate often did not reach 30 times per minute, and most of the patients GCS was more than 8. Analyzing the significance of CURB65 in fast evaluation in severity of pneumonia, some may question the importance/significanse of Urea in this scale. Authors suggest to add laboratorial markers, which potentially can be more significant in evaluation of severity of pneumonia, like White Cell Count and Serum lactate levels. We did not find statistically reliable correlation between CRP and outcome which is similar to other research data, but PCT is a very good prognostic marker (Heper, 2006). This study can not define the specific serum lactate border value to evaluate which patients should be admitted to ICU. This border value could be lower than it is used to be defined, even if the first lactate measurement is $>2 \mathrm{mmol} / \mathrm{L}$. In the future it would be worthy to analyze dynamic lactate levels and their correlation with outcome and to make a prospective study that would allow us to analyze all the therapeutic, toxic, pre hospital and other factors that could influence lactate levels in each specific case. An also it would be useful to explore also oxygenation and saturation in prospective study.

\section{CONCLUSIONS}

1. Initial serum lactate level has a significant prognostic value in the first $24 \mathrm{~h}$.

2. Initial serum lactate level $>3 \mathrm{mmol} / \mathrm{L}$ is related with increased risk of mortality rate for patients with community-acquired pneumonia.

3. All lactate measurements ( $\mathrm{LAC}_{1}, \mathrm{LAC}_{2}, \mathrm{LAC}_{3}, \mathrm{LAC}_{4}$ ) has a similar prognostic ability with APACHE II and PCT scales and they are better than CRP and CURB65.

4. Early lactate level measurement before admission to ICU can help stratify patients with high risk of mortality.

\section{Conflict of interest: None}

\section{REFERENCES}

1. Bernardin, G., Pradier, C., Tiger, F., et al. Blood pressure and arterial lactate level are early indicators of shortterm survival in human septic shock// Intensive Care Medicine, 1996; 22:17-25

2. Berry, M. N. The liver and lactic acidosis// Proc. Royal Society of Medicine, 1967; 60: 1260-2

3. Bersten, A.D., Soni, N. Oh's intensive care manual. 6th ed. - Philadelphia: Butterworth Heinemann Elsvier 2009. - Pp. 145.-147

4. Broder, G., Weil, M.H. Excess lactate: an index of reversibility of shock in human patients// Science, 1964; 143: 1457-9

5. Cady, L. D., Weil, M. W., Afifi, A. A., et al. Quantitation of severity of critical illness with special reference to blood lactate//Critical Care Medicine, 1973; 1:75-80

6. Chertoff, J., Chisum, M., Garcia, B., Lascano, J. Lactate kinetics in sepsis and septic shock: a review of the literature and rationale for further research// Journal of Intensive Care, 2015; 3:39

7. Dellinger, R. P., Levy, M. M., Rhodes, A., et al. Surviving Sepsis Campaign: International Guidelines for Management of Severe Sepsis and Septic Shock: 2012// CriticalCare Medicine, 2013; 41: 580-595

8. Gillet, Y., Bertrand, I., Vanhems, P., et al. Association between Staphylococcus aureus strains carrying gene for Panton-Valentine leukocidin and highly lethal necrotising pneumonia in young immunocompetent patients//Lancet, 2002; 359: $753-759$ 
9. Gunnerson, K.J., Saul, M., He, S. et al. Lactate versus nonlactate metabolic acidosis: a retrospective outcome evaluation of critically ill patients//Critical Care Medicine, 2006; 10(1): R22

10. Gutierrez, F., Masia, M., Rodriguez, J. C., et al. M. Epidemiology of community-acquired pneumonia in adult patients at the dawn of the 21 st century: a prospective study on the Mediterranean coast of Spain. Clinical Microbiology and Infectology, 2005; 11: 788-800

11. Gutierrez, G., Wulf, M. E. Lactic acidosis in sepsis: another commentary// Critical Care Medicine, 2005; 33: 2420-2

12. Heper, Y., Akal, E. H., Mistik, R., et al. Evaluation of serum C-reactive protein, procalcitonin, tumor necrosis factor alpha, and interleukin-10 levels as diagnostic and prognostic parameters in patients with communityacquired sepsis, severe sepsis, and septic shock// European Journal ofClinical Microbiology and Infectious Diseases, 2006 25:481-491

13. Jansen, T. C., Bommel, J., Schoonderbeek, J.,et al. Early Lactate-Guided Therapy in Intensive Care Unit Patients// American Journal of Respiratory and Critical Care Medicine, 2010; 182: 752-761

14. Jat, K. N., Jhamb, U., Gupta, V. K. Serum lactate levels as the predictorof outcome in pediatric septic shock// Indian Journalof Critical Care Medicine, 2011; 15: 102-7

15. Knaus, W. A., Draper, E. A., Wagner, D. P., et al. APACHE II: a severity of disease classification system// Critical Care Medicine, 1985; 13: 818-29

16. Loke, Y. K., Kwok, C. S., Niruban, A., Myint, P. $\mathrm{K}$. Value of severity scales in predicting mortality from community-acquired pneumonia: systematic rewiev and meta-analysis// Thorax, 2010; 65: 884890

17. Lozano, R., Naghavi, N., Foreman, K.,et al. Global and regional mortality from 235causesof death for 20 age groups in 1990 and 2010: a systematic analysis for the Global Burden of Disease Study 2010// Lancet, 2012; 380 (9859): 2095-128

18. Members of the American College of Chest Physicians/Society of Critical Care Medicine Consensus Conference Committee (1992). Definitions for sepsis and organ failure and guidelines for the use of innovative therapies in sepsis// Critical Care Medicine, 1992; 20: 864-874

19. Mikkelsen, M. E., Miltiades, A. N., Gaieski, D. F., et al. Serum lactate is associated with mortality in severe sepsis independent of organ failure and shock// Critical Care Medicine, 2009; 37: 16701677

20. Moine, P., Vercken, J. B., Chevret, S., et al. Severe Community-Acquired Pneumonia: Etiology, Epidemiology, Prognosis Factors//Chest, 1994; 105:1487-1495
21. Neil, A. M., Martin, I. R., Weir, R. et al. Community acquired pneumonia: aetiology and usefullness of severity criteria on admission// Thorax, 1996; 51: $1010-6$

22. Odom, S. R. Llactate clearance as a predictor of mortality//The Journal of Tauma and Acute Care Surgery, 2014; 77: 183-4

23. Peake, L. S., Denaley, A., Bailey, M., et al (ARISE Investigators and the ANZICS Clinical Trials Group). Goal-Directed Resuscitation for Patients with Early Septic Shock//New England Journal of Medicine, 2014; 371: 1496-506

24. Peake, S. L., et al. Goal-directed resuscitation for patients with early septic shock. N Engl J Med, 2014; 371(16): 1496-506

25. Richards, G.,Levy, H., Laterre, P. F., Feldman, C., Woodward, B., Bates, B. M., Qualy, R. L. CURB65, PSI, and APACHE II to assess mortality risk in patients with severe sepsis and community acquired pneumonia in PROWESS//Journal of Intensive Care Medicine, 2011 ; 26(1): 34-40

26. Rivers, E., Nguyen, B., Havstad, S. et al. Early goaldirected therapy in treatment of severe sepsis and septic shock//New England Journal of Medicine, $2001 ; 345: 1368-77$

27. Shapiro, N., Howell, M. D., Talmor, D., et al. Serum lactate as a predictor of mortality in emergency department patients with infection// Annals of Emergency Medicine, 2005; 45(5):524-8

28. Sligl, W. I., Marrie, J. T. Severe CommunityAcquired Pneumonia// Critical Care Clinics, 2013; 29: 563-601

29. Smith, I., Kumar, P., Molly, S., et al. Base excess and lactate as prognostic indicators for patients admitted to intensive care// Intensive Care Medicine, 2001; 27:74-83

30. Suistomaa, M., Ruokonen, E., Kari, A., et al. Timepattern of lactate and lactate to pyruvate ratio in the first 24 hours of intensive care emergency admissions// Shock, 2000; 14(1): 8-12

31. Wiemken, L. T., Peyrani, P., Ramirez, J. A. Global Changes in the Epidemiology of CommunityAcquired Pneumonia// Semin of Respiratory Critical Care Medicine, 2012; 33: 213-219
Address:
Darta Ose
Latvija, Saulkrasti,
cirulu street 3, LV-2160
e-mail: darta.ose@gmail.com 